\title{
Diffusion of concentrated neutral hard-sphere colloidal suspensions
}

\author{
R. Verberg and I. M. de Schepper \\ IRI Delft University of Technology, 2629 JB Delft, The Netherlands \\ E. G. D. Cohen \\ The Rockefeller University, New York, New York 10021 \\ (Received 6 July 1999; revised manuscript received 30 September 1999)
}

\begin{abstract}
We evaluate theoretical expressions for the long-time wave-number-dependent self-diffusion and collective diffusion coefficients $D_{S}^{L}(k, \phi)$ and $D^{L}(k, \phi)$, respectively, as a function of volume fraction $\phi$ and wave vector $k$ for neutral monodisperse hard-sphere colloidal suspensions over the entire fluid range. The theory is based on the Smoluchowski equation with mean-field-like hydrodynamic interactions, cage diffusion, and is free of adjustable parameters. The basic physical mechanisms underlying our formulas are discussed and the results are compared with recent experimental results by Segrè and Pusey [Phys. Rev. Lett. 77, 771 (1996)].
\end{abstract}

PACS number(s): 82.70.Dd, 83.10.Ff

\section{INTRODUCTION}

In previous papers we presented a molecular theory for the dynamic (frequency-dependent) and the long-time macroscopic (Newtonian) viscosity [2-4] and for the long-time macroscopic self-diffusion coefficient $[4,5]$ of concentrated neutral hard-sphere-like colloidal suspensions. This theory was based on the idea, first developed for concentrated molecular fluids using kinetic theory [6-8], that for high concentration colloidal suspensions (volume fractions $\phi>0.35$ ), the dominant physical process is a short-time cage-diffusion process. Here one uses that for $\phi>0.35$ each colloidal particle finds itself in a cage (of about the size of the hardsphere diameter $\sigma$ ) formed by its neighbors, which makes the escape from the cage difficult. This leads to a slow relaxation of the particle displacements involved in collective density fluctuations back to their equilibrium positions. This relaxation is particularly slow for density fluctuations of wave number $k=k^{*} \approx 2 \pi / \sigma$, since the equilibrium static structure factor $S(k, \phi)$ has a very pronounced maximum near $k$ $=k^{*}$, implying a great amount of ordering in the suspension, which greatly inhibits the escape of particles from their cages. This physical picture has been confirmed by light scattering experiments on concentrated colloidal suspensions as well as neutron scattering experiments of atomic liquids.

Here we apply the same idea to the long-time wavenumber-dependent self-diffusion and collective diffusion coefficients, $D_{S}^{L}(k, \phi)$ and $D^{L}(k, \phi)$, respectively, where $D_{S}^{L}(\phi)=D_{S}^{L}(k=0, \phi)$, the long-time macroscopic selfdiffusion coefficient [9]. We show that good agreement with experiment can be obtained by using cage diffusion as the dominant physical process determining both the short-time as well as the long-time diffusive behavior at high concentrations. In particular, the long-time wave-number-dependent collective (self-) diffusion coefficient can be written in good approximation as a product of two contributions. First, a short-time contribution $D^{S}(k, \phi)\left[D_{S}^{S}(\phi)\right]$, directly related to the initial slope of the equilibrium intermediate (self-) scattering function $F(k, \phi, t)\left[F_{S}(k, \phi, t)\right]$ as a function of $t$, in which a particle rattles inside the cage, formed around it by its nearest neighbors. Second, a long-time contribution in which a particle moves from cage to cage. This long-time contribution is calculated explicitly from the Smoluchowski equation using the mode-mode coupling approximation (MMCA) with hydrodynamical interactions incorporated in a mean-field-like manner. In order to obtain $D^{L}(k, \phi)$ explicitly one needs the three-particle correlation function. It is shown that several suggestions in the literature lead to very different results, but that the approximation proposed by Jackson and Feenberg [10] appears to work well.

The past years have seen a considerable effort in this field of study dating back to the first theoretical expressions for $D_{S}^{L}(k, \phi)$ and $D^{L}(k, \phi)$, obtained directly from the FokkerPlanck equation [11] and later shown to be equal to the results obtained from the Smoluchowski equation [12]. However, those results neglected hydrodynamic interactions altogether and are consequently only valid at very low corevolume fractions. Furthermore, Hess and Klein [11] used an unphysical approximation for the three-particle correlation function to compare their expression for $D^{L}(k, \phi)$ with experimental results, a point further discussed below. More recently, Szamel and Löwen [13], Wagner [14], and Baur et al. [15] extended those early results, but again neglect hydrodynamic interactions completely. Szamel and Löwen used the Jackson and Feenberg [10] approximation for the threeparticle correlation function and investigated the glass transition of a colloidal suspension [13]. Wagner used a selfconsistent viscoelastic relaxation model and compared his theory with experimental results obtained for strongly correlated charged suspensions [14]. He also compared two approximations for the three-particle correlation function, i.e., the Hess and Klein [11] approximation and the Jackson and Feenberg [10] approximation, a point also discussed below. Baur et al. also used the Jackson and Feenberg [10] approximation for the three-particle correlation function, but included in first approximation the effect of polydispersity on the so-called nonexponentiality factor [15]. Very recently, further progress has been made by incorporating far-field hydrodynamic interactions [16] and extending the results to polydisperse suspensions and colloidal mixtures $[17,18]$. However, although clearly a considerable improvement for strongly interacting charged suspensions or neutral suspensions at a low volume fraction, far-field hydrodynamic inter- 
actions are certainly not suitable for concentrated neutral suspensions. Since the extension to colloidal mixtures is beyond the scope of this paper and the results reduce to the results of Nägele and Baur [16] for one-component suspensions, no further reference to these papers is made. As far as we know, the only results valid for concentrated neutral suspensions are those obtained by Medina-Noyola [19], who calculated $D_{S}^{L}(k, \phi)$ by solving approximately the Langevin equation for the velocity of a tracer particle and those obtained by Banchio et al. [20], who presented theoretical results for $D^{L}\left(k=k^{*}, \phi\right)$ and $D_{S}^{L}(\phi)$ as well as for the Newtonian viscosity, results that are further discussed below.

The organization of this paper is as follows. We start with the theoretical derivation of our results in Sec. II. In Sec. III we consider the short-time self-diffusion and collective diffusion coefficients $D_{S}^{S}(\phi)$ and $D^{S}(k, \phi)$, respectively, where short times refer to the region $\tau_{B} \sim t \ll \tau_{P}$. Here $\tau_{B}=m / \zeta_{0}$ $\sim 1 \mathrm{~ns}$, is the Brownian time in which the initial velocity of a particle of mass $m$ relaxes to equilibrium and $\tau_{P}$ $=\sigma^{2} / 4 D_{0} \sim 1 \mathrm{~ms}$ is the Péclet or interaction time, characteristic for free particle diffusion over a distance equal to its own radius. The friction and self-diffusion coefficient of an isolated Brownian particle, $\zeta_{0}$ and $D_{0}$, respectively, are related through the Einstein relation $\zeta_{0}=k_{B} T / D_{0}$, with $k_{B}$ Boltzmann's constant and $T$ the temperature. In Sec. IV the long-time $\left(t \gg \tau_{P}\right)$ wave-number-dependent self-diffusion coefficient $D_{S}^{L}(k, \phi)$ is evaluated and its limit for $k=0$, i.e., the macroscopic self-diffusion coefficient $D_{S}^{L}(\phi)$, is compared with experimental results. In Sec. V the long-time $(t$ $\gg \tau_{P}$ ) wave-number-dependent collective diffusion coefficient $D^{L}(k, \phi)$ is evaluated for different approximations of the three-particle correlation function and compared with the recent experimental results of Segrè and Pusey [1]. We conclude with a discussion of the main results.

\section{THEORY}

The diffusive behavior in concentrated colloidal suspensions is connected to the decay of collective density fluctuations, described by the the wave number $k$ and time $t$ dependent equilibrium intermediate scattering function $F(k, \phi, t)$, defined by

$$
F(k, \phi, t)=\frac{1}{N}\left\langle\delta n(-\mathbf{k}) e^{\hat{\Omega} t} \delta n(\mathbf{k})\right\rangle,
$$

where $\delta n(\mathbf{k})$ is a plane wave collective density fluctuation mode with wave vector $\mathbf{k}$, i.e.,

$$
\delta n(\mathbf{k})=\sum_{i=1}^{N} e^{-i \mathbf{k} \cdot \mathbf{r}_{i}}-\left\langle\sum_{i=1}^{N} e^{-i \mathbf{k} \cdot \mathbf{r}_{i}}\right\rangle,
$$

with $\mathbf{r}_{i}$ the position of particle $i$ at time $t=0$. The brackets \langle\rangle denote the equilibrium ensemble average with the canonical distribution function and $\hat{\Omega}$ is the adjoint Smoluchowski operator describing the time evolution of the system (e.g., Ref. $[21,22])$

$$
\hat{\Omega}=\sum_{i, j=1}^{N}\left\{\boldsymbol{\nabla}_{i}+\beta \mathbf{F}_{i}(\Gamma)\right\} \cdot \mathbf{D}_{i j}(\Gamma) \cdot \nabla_{j}
$$

Here $\boldsymbol{\nabla}_{i}=\partial / \partial \mathbf{r}_{i}, \beta=1 / k_{B} T, \mathbf{F}_{i}(\Gamma)=-\nabla_{i} U(\Gamma)$ is the force on particle $i$ due to direct interactions with all other particles in the configuration space $\Gamma=\left(\mathbf{r}_{1}, \ldots, \mathbf{r}_{N}\right)$ and $\mathbf{D}_{i j}(\Gamma)$ is the diffusion tensor which incorporates hydrodynamic interactions.

The corresponding definition of the equilibrium intermediate self-scattering function $F_{S}(k, \phi, t)$ is

$$
F_{S}(k, \phi, t)=\left\langle\delta n_{1}(-\mathbf{k}) e^{\hat{\Omega} t} \delta n_{1}(\mathbf{k})\right\rangle
$$

with $\delta n_{1}(\mathbf{k})$ the one-particle density fluctuation mode

$$
\delta n_{1}(\mathbf{k})=e^{-i \mathbf{k} \cdot \mathbf{r}_{1}}-\left\langle e^{-i \mathbf{k} \cdot \mathbf{r}_{1}}\right\rangle .
$$

Here $\mathbf{r}_{1}$ is the tagged particle's position at time $t=0$. We note that from partial integration one finds the relation

$$
\left\langle\mathbf{F}_{i}(\Gamma) f(\Gamma)\right\rangle=-\beta\left\langle\nabla_{i} f(\Gamma)\right\rangle
$$

for any function $f(\Gamma)$, a result that is frequently used below.

\section{A. Short-time self-diffusion and collective diffusion}

First, we consider the short-time self-diffusion and collective diffusion coefficients, where short times refer to the region $\tau_{B} \sim t \ll \tau_{P}$. For short times the equilibrium intermediate scattering function behaves as

$$
F(k, \phi, t)=S(k, \phi) e^{-k^{2} D^{S}(k, \phi) t},
$$

while the analogous expression for $F_{S}(k, \phi, t)$ is

$$
F_{S}(k, \phi, t)=e^{-k^{2} D_{S}^{S}(\phi) t}
$$

Here $S(k, \phi)=F(k, \phi, t=0)$ and $D_{S}^{S}(\phi)$ and $D^{S}(k, \phi)$ are the short-time self-diffusion and collective diffusion coefficients, respectively, which can be expressed in terms of $k$-dependent averages over the diffusion tensor $\mathbf{D}_{i j}(\Gamma)$ (e.g., Refs. [21,22]), but a simple explicit expression that fully incorporates the complicated many-body hydrodynamic interactions for volume fractions $\phi>0.4$ is still lacking. Therefore, we propose alternative explicit expressions for $D_{S}^{S}(\phi)$ and $D^{S}(k, \phi)$, based on the analogy between neutral hardsphere colloidal suspensions and molecular fluids and compare them with experimental results in Sec. III.

\section{B. Long-time collective diffusion}

Next we consider the long-time collective diffusion coefficient $D^{L}(k, \phi)$, defined in terms of the Laplace transform $\widetilde{F}(k, \phi, z)=\int_{0}^{\infty} d t \exp (-z t) F(k, \phi, t)$ of $F(k, \phi, t)$ for $z=0$, leading to (e.g., Refs. [21,22])

$$
D^{L}(k, \phi)=S(k, \phi) / \int_{0}^{\infty} d t k^{2} F(k, \phi, t) .
$$

To develop a theory for $D^{L}(k, \phi)$ we start from Eqs. (1) and (9) and replace, like Brady [23], the adjoint Smoluchowski operator $\hat{\Omega}$ with its corresponding mean-field expression 


$$
\hat{\Omega}_{M F}=D_{S}^{S}(\phi) \sum_{i=1}^{N}\left\{\boldsymbol{\nabla}_{i}+\beta \mathbf{F}_{i}(\Gamma)\right\} \cdot \boldsymbol{\nabla}_{i},
$$

i.e., by approximating the diffusion tensor $\mathbf{D}_{i j}(\Gamma)$ in Eq. (3) by its "mean-field" average $\left\langle\mathbf{D}_{i j}(\Gamma)\right\rangle=\delta_{i j} 1 D_{S}^{S}(\phi)$. Then, applying standard projection operator techniques to Eq. (1), and generalizing the procedures developed by Cichocki and Hess [12] for low concentrations to high concentrations, we obtain an expression for $D^{L}(k, \phi)$ for all $\phi$, i.e.,

$$
D^{L}(k, \phi)=\frac{D_{S}^{S}(\phi) / S(k, \phi)}{1+\tilde{M}(k, \phi) /\left[k^{2} D_{S}^{S}(\phi)\right]},
$$

with $\tilde{M}(k, \phi)=\int_{0}^{\infty} d t M(k, \phi, t)$. Here $\tilde{M}(k, \phi)$ is directly proportional to the generalized $k$-dependent longitudinal viscosity [12] and $M(k, \phi, t)$ is the collective longitudinal stress time-autocorrelation function,

$$
M(k, \phi, t)=\frac{\left[\beta k^{2} D_{S}^{S}(\phi)\right]^{2}}{N}\left\langle\sigma_{\|}(-\mathbf{k}) e^{\hat{\Omega}_{M F}^{i r r} t} \sigma_{\|}(\mathbf{k})\right\rangle,
$$

where the microscopic longitudinal stress is given by

$$
\sigma_{\|}(\mathbf{k})=-\sum_{i=1}^{N}\left\{\frac{i \mathbf{k} \cdot \mathbf{F}_{i}}{k^{2}}+k_{B} T \frac{S(k, \phi)-1}{S(k, \phi)}\right\} e^{-i \mathbf{k} \cdot \mathbf{r}_{i}},
$$

and $\widehat{\Omega}_{M F}^{i r}$ is the mean-field expression for the irreducible adjoint Smoluchowski operator. $\hat{\Omega}_{M F}^{i r r}$ can be obtained from the result of Cichocki and Hess [12], by using the mean-field approximation for the diffusion tensor discussed below Eq. (10). The irreducible Smolochowski operator keeps $\sigma_{\|}(\mathbf{k})$ orthogonal to all single collective density fluctuation modes when it evolves in time. Therefore, the decay of $M(k, \phi, t)$ will be determined in first approximation by the decay of two coupled collective density fluctuation modes.

To calculate this coupled decay contribution to $M(k, \phi, t)$ of Eq. (12) we apply the mode coupling theory and introduce the projection operator $\hat{P}_{2}$ on bilinear products of two collective density fluctuations as in Refs. [11,22], i.e.,

$$
\hat{P}_{2}=\sum_{\mathbf{k}_{1}, \mathbf{k}_{2}} \frac{\left|\delta n\left(\mathbf{k}_{1}\right) \delta n\left(\mathbf{k}_{2}\right)\right\rangle\left\langle\delta n\left(\mathbf{k}_{1}\right) \delta n\left(\mathbf{k}_{2}\right)\right|}{2 N^{2} S\left(k_{1}, \phi\right) S\left(k_{2}, \phi\right)} .
$$

We restrict the action of $\exp \left(\hat{\Omega}_{M F}^{i r r} t\right)$ in Eq. (12) to the space of products of density fluctuations only, i.e., we write

$$
e^{\hat{\Omega}_{M F}^{i r r} t} \approx \hat{P}_{2} e^{\hat{\Omega}_{M F}^{i r r} t} \hat{P}_{2} .
$$

From Eqs. (12), (14), and (15) one finds then for $M(k, \phi, t)$,

$$
\begin{aligned}
M(k, \phi, t)= & \frac{1}{16 \pi^{3} n} \int d \mathbf{q}\left[\frac{V(\mathbf{k}, \mathbf{q})}{S(q, \phi) S(|\mathbf{k}-\mathbf{q}|, \phi)}\right]^{2} \\
& \times F(q, \phi, t) F(|\mathbf{k}-\mathbf{q}|, \phi, t) .
\end{aligned}
$$

A similar expression for $M(k, \phi, t)$ has been derived before by neglecting hydrodynamic interactions altogether, cf. Refs. $[11,14]$ (based on the Fokker-Planck equation) and Refs. $[13,15]$ (based on the Smoluchowski equation) and recently independently from us by using a far-field approximation for the diffusion tensor [16]. Those different approximations only result in different expressions for the vertex function $V(\mathbf{k , q})$. In the mean-field approximation the vertex function, representing the strength of the coupling between the microscopic longitudinal stress $\sigma_{\|}(\mathbf{k})$ and the bilinear products $\delta n(\mathbf{q}) \delta n(\mathbf{k}-\mathbf{q})$, is given by

$$
V(\mathbf{k}, \mathbf{q})=\frac{\left[\beta k^{2} D_{S}^{S}(\phi)\right]}{N}\left\langle\sigma_{\|}(-\mathbf{k}) \delta n(\mathbf{q}) \delta n(\mathbf{k}-\mathbf{q})\right\rangle .
$$

Using Eq. (13) for $\sigma_{\|}(\mathbf{k})$ and Eq. (6) yields

$$
\begin{aligned}
V(\mathbf{k}, \mathbf{q})= & D_{S}^{S}(\phi) \mathbf{k} \cdot\left\{\frac{\mathbf{k} T(\mathbf{k}, q)}{S(k, \phi)}-\mathbf{q} S(|\mathbf{k}-\mathbf{q}|, \phi)\right. \\
& -(\mathbf{k}-\mathbf{q}) S(q, \phi)\}
\end{aligned}
$$

where

$$
T(\mathbf{k}, \mathbf{q})=\frac{1}{N}\langle\delta n(-\mathbf{k}) \delta n(\mathbf{q}) \delta n(\mathbf{k}-\mathbf{q})\rangle
$$

is a wave-number-dependent three-particle correlation function. One easily shows that $T(\mathbf{k}, \mathbf{q})=S(k, \phi)$ ( $k$ finite, $q$ large $), \quad T(\mathbf{k}, \mathbf{q})=S(q, \phi) \quad(k$ large, $q$ finite $)$ and $T(\mathbf{k}, \mathbf{q})$ $=S(|\mathbf{k}-\mathbf{q}|, \phi) \quad(k$ large, $q$ large, and $|\mathbf{k}-\mathbf{q}|$ finite $)$. For explicit calculations we use different approximations introduced in the literature, which all satisfy these limiting properties. We then evaluate $M(k, \phi, t)$ of Eq. (16) for all times using the short-time expression (7) for $F(|\mathbf{k}-\mathbf{q}|, \phi, t)$ and $F(q, \phi, t)$. In Sec. V, we compare $D^{L}(k, \phi)$ for different approximations of the three-particle correlation function with experimental results.

\section{Long-time self-diffusion}

Finally, we consider the long-time self-diffusion coefficient $D^{S}(k, \phi)$. Since the derivation is essentially the same as that for the long-time collective diffusion coefficient presented above, we only give the final results. The long-time wave-number-dependent self-diffusion coefficient $D_{S}^{L}(k, \phi)$ can be obtained from the equilibrium intermediate selfscattering function via (e.g., Refs. [21,22])

$$
D_{S}^{L}(k, \phi)=1 / \int_{0}^{\infty} d t k^{2} F_{S}(k, \phi, t) .
$$

Starting then from Eqs. (4) and (20), replacing $\hat{\Omega}$ by $\hat{\Omega}_{M F}$ [cf. Eq. (10)] and proceeding as before in Eqs. (11) to (17) we obtain

$$
D_{S}^{L}(k, \phi)=\frac{D_{S}^{S}(\phi)}{1+\widetilde{M}_{S}(k, \phi) /\left[k^{2} D_{S}^{S}(\phi)\right]},
$$

with $\widetilde{M}_{S}(k, \phi)=\int_{0}^{\infty} d t M_{S}(k, \phi, t)$, where 


$$
\begin{aligned}
M_{S}(k, \phi, t)= & \frac{1}{8 \pi^{3} n} \int d \mathbf{q}\left[\frac{V_{S}(\mathbf{k}, \mathbf{q})}{S(q, \phi)}\right]^{2} \\
& \times F(q, \phi, t) F_{S}(|\mathbf{k}-\mathbf{q}|, \phi, t),
\end{aligned}
$$

with the self-vertex function $V_{S}(\mathbf{k}, \mathbf{q})$ given by

$$
V_{S}(\mathbf{k}, \mathbf{q})=D_{S}^{S}(\phi)(\mathbf{k} \cdot \mathbf{q})[S(q, \phi)-1] .
$$

Expressions similar to Eq. (22) have been obtained before by neglecting hydrodynamic interactions altogether $[11,15]$ and by using a far-field approximation for the diffusion tensor [16], resulting in different expressions for the self-vertex function $V_{S}(\mathbf{k , q})$.

We evaluate $M_{S}(k, \phi, t)$ for all times in terms of the short-time expressions for $F(q, \phi, t)$ of Eq. (7) and $F_{S}(\mathbf{k}$ $-\mathbf{q}, \phi, t)$ of Eq. (8). We remark that the decay of $M_{S}(k, \phi, t)$ is determined in first approximation by the decay of one collective density fluctuation mode and one one-particle density fluctuation mode. Therefore, the operator $\hat{P}_{2}$ projects for self-diffusion on the bilinear product of a collective density fluctuation and a one-particle density fluctuation mode (cf. Refs. $[11,22])$. This results in the appearance of the equilibrium intermediate self-scattering function $F_{S}(|\mathbf{k}-\mathbf{q}|, \phi, t)$ in Eq. (22) [cf. Eq. (16)].

\section{SHORT-TIME SELF-DIFFUSION AND COLLECTIVE DIFFUSION}

At short times $\tau_{B} \sim t \ll \tau_{P}$ the dominant physical process involves the interaction of the Brownian particles with the fluid and with each other through the fluid, the so-called hydrodynamic interactions. These hydrodynamic interactions are complicated many-particle interactions for which explicit evaluations exist only in limiting cases. To date there are only exact results for $D_{S}^{S}(\phi)$ and $D^{S}(k, \phi)$ for two isolated hard-sphere particles based on series expansion of the hydrodynamic functions in powers of the inverse distance between the centers of the two particles $[24,25]$ (a recent overview is presented by Dhont [22]). These results are valid up to second order in the volume fraction $\phi$, thus clearly restricting the applicability to low volume fractions. Approximate evaluations were made by Beenakker and Mazur [26], by considering the hydrodynamical motion of one hard-sphere particle in a stationary field due to all other particles at rest, which restricts the applicability effectively to short times $\tau_{B} \sim t \ll \tau_{P}$. Using a density cluster expansion technique they obtained results for the self-diffusion and collective diffusion coefficient up to intermediate volume fractions $\phi \approx 0.4$. More recently, accurate numerical results were obtained for volume fraction up to $\phi=0.45$, based on multipole expansion for the hydrodynamic interactions and lubrication theory for the singular forces near contact [27].

Up to now it seems that no explicit results for the transport properties of concentrated colloidal suspensions, i.e., for volume fractions $\phi>0.40$, which fully take into account the many-particle hydrodynamic interactions, are available in the literature. Therefore, we propose to use a short-time collective diffusion coefficient $D^{S}(k, \phi)$, which is physically interpreted in terms of the characteristic cage-diffusion coefficient $D_{C}(k, \phi)$, in analogy with the result for concentrated

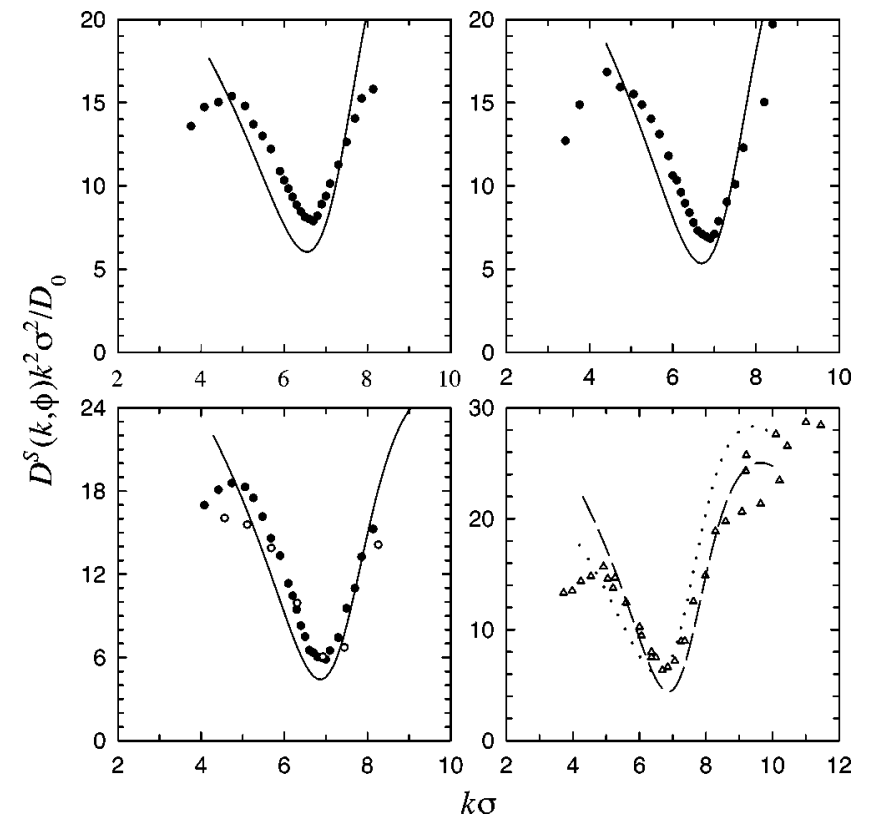

FIG. 1. Reduced short-time collective diffusion coefficient $D^{S}(k, \phi) k^{2} \sigma^{2} / D_{0}$ as a function of $k \sigma$. The datapoints are experimental results for suspensions of neutral polymethylmethacrylate (PMMA) spheres obtained by [41] $(\bigcirc),[42](\bigcirc)$, and [30] $(\triangle)$. The solid line represents Eq. (24) for the volume fractions $\phi=0.443$ (a), 0.465 (b), and 0.494 (c). In (d) the dotted line corresponds to a surface layer of $9 \mathrm{~nm}(\phi=0.44)$ and the dashed line to one of 14 $\mathrm{nm}(\phi=0.49)[30]$.

molecular fluids developed from basic kinetic theory $[6,7]$, i.e.,

$$
D^{S}(k, \phi) \approx D_{C}(k, \phi)=\frac{D_{S}^{S}(\phi) d(k)}{S(k, \phi)}
$$

Here $S(k, \phi)$ is the equilibrium static structure factor and $d(k)=\left[1-j_{0}(k \sigma)+2 j_{2}(k \sigma)\right]^{-1}$, a combination of spherical Bessel functions $j_{l}(k)$ of the order $l$, with $\sigma$ the diameter of the Brownian particle.

As said, Eq. (24) is an adaptation of a very similar formula for the cage-diffusion coefficient in concentrated molecular fluids, where the short-time Enskog diffusion coefficient $D_{E}(\phi)$ is replaced by the short-time self-diffusion coefficient $D_{S}^{S}(\phi)$ of a colloidal suspension. Consistent with this analogy between $D_{E}(\phi)$ and $D_{S}^{S}(\phi)$, Cohen and de Schepper [28] suggested to use for the short-time selfdiffusion coefficient in concentrated colloidal suspensions the (phenomenological) expression

$$
D_{S}^{S}(\phi)=\frac{D_{0}}{\chi(\phi)},
$$

where the Boltzmann free diffusion coefficient $D_{B}(\phi)$ is replaced by its colloidal equivalence, i.e., by the StokesEinstein diffusion coefficient $D_{0}$ of an isolated Brownian particle. Here $\chi(\phi)=g(r=\sigma, \phi)$, the equilibrium pair distribution function of two hard-sphere particles of diameter $\sigma$ at contact.

Equations (24) and (25) are compared with experimental results on neutral colloidal suspensions in Figs. 1 and 2, respectively. Here the Carnahan-Starling approximation 


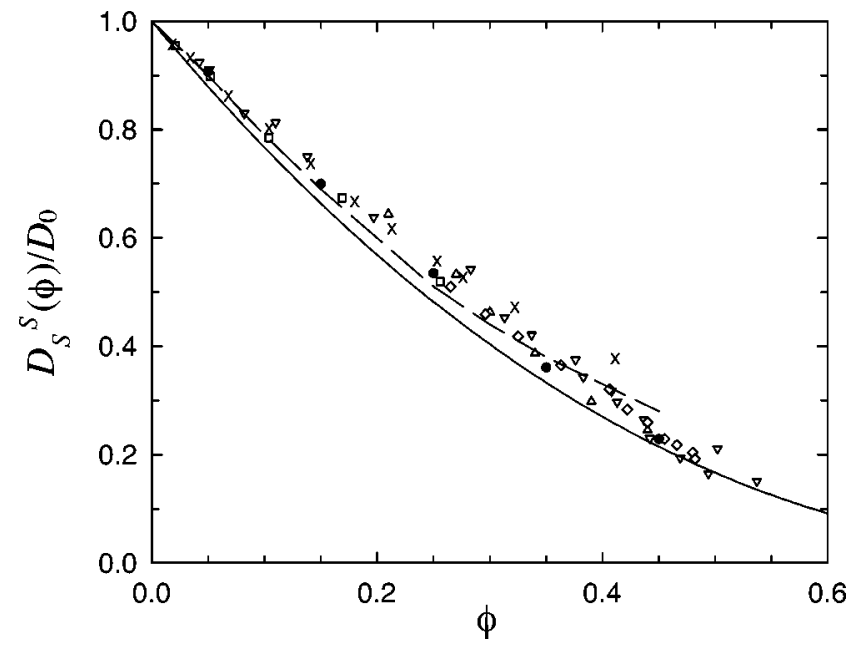

FIG. 2. Relative short-time self-diffusion coefficient $D_{S}^{S}(\phi) / D_{0}$ as a function of the volume fraction $\phi$. The datapoints are experimental results of polystyrene spheres in water obtained by [43] ( $\square)$, neutral PMMA spheres in organic solvents obtained by $[36](\nabla)$, [44] $(\triangle)$, and [41] $(\diamond)$, and of double coated silica spheres in tetrahydrofufurylalcohol [45] $(\times)$. The solid dots are computer simulation results [46]. The solid line represents Eq. (25) and the dashed line corresponds to the result of Beenakker and Mazur [26].

$\chi(\phi)=(1-0.5 \phi) /(1-\phi)^{3}$ is used and the static structure factor is calculated in the Percus-Yevick approximation, with a correction discussed by Henderson and Grundke [29]. We find systematically a lower result for $D^{S}(k, \phi) k^{2} \sigma^{2} / D_{0}$, as compared with the experimental data (cf. Fig. 1). This is consistent with the fact that our value for $D^{S}(k, \phi)$ at $k$ $=k^{*}$ is about $20 \%$ smaller than the value obtained by using the semiempirical result for the hydrodynamic function $H\left(k^{*}, \phi\right)=1-1.35 \phi=D^{S}\left(k^{*}, \phi\right) S\left(k^{*}, \phi\right) / D_{0}[20]$. The underestimation of the value for $D^{S}(k, \phi)$ at $k=k^{*}$ is most probably a result of the neglect of the polydispersity on the value of the maximum of the $S(k, \phi)$, since polydispersity lowers the first peak of the $S(k, \phi)$. The discrepancy can also be related to small inaccuracies in the determination of the effective hard-sphere diameter $\sigma$, as is illustrated in Fig. 1(d). Here $D^{S}(k, \phi) k^{2} \sigma^{2} / D_{0}$ is calculated for two values of $\sigma$, corresponding to a surface layer of $9 \mathrm{~nm}$ and $14 \mathrm{~nm}$ [30]. The uncertainty in the determination of the volume fraction also causes difficulty in interpreting quantitatively the various experimental results [31,32].

In Fig. 2, the result of Beenakker and Mazur [26], who obtained $D_{S}^{S}(\phi)$ for volume fractions up to $\phi=0.45$, is also presented. Considering the small deviations between their result and that of Eq. (25) and our primary interest in high volume fractions $\phi>0.40$, Eq. (25) seems to be able to describe the experimental data over the entire fluid range, i.e., for volume fractions $0<\phi<0.55$. We note that our approximation becomes increasingly better for high volume fractions $\phi>0.40$.

\section{LONG-TIME SELF-DIFFUSION}

In Sec. II we derived an expression [cf. Eq. (21)] for the long-time wave-number-dependent self-diffusion coefficient $D_{S}^{L}(k, \phi)$ in terms of $\widetilde{M}_{S}(k, \phi)=\int_{0}^{\infty} d t M_{S}(k, \phi, t)$, with
$M_{S}(k, \phi, t)$ given by Eq. (22). Restricting ourself to first order mode-coupling theory implies that in Eq. (22) the shorttime expressions (7) for $F(k, \phi, t)$ and (8) for $F_{S}(k, \phi, t)$ can be used. This restriction has already been discussed in the application of the MMCA to the diffusion and the viscosity of dense molecular fluids and to the viscosity of concentrated colloidal suspensions and seems valid for volume fractions in the fluid range $[4,33]$.

Then, the explicit result for the long-time wave-numberdependent self-diffusion coefficient $D_{S}^{L}(k, \phi)$ is given by

$$
\begin{aligned}
\frac{D_{S}^{S}(\phi)}{D_{S}^{L}(k, \phi)}= & 1+\frac{D_{S}^{S}(\phi)}{8 \pi^{3} n} \\
& \times \int d \mathbf{q} \frac{\{\hat{\mathbf{k}} \cdot \mathbf{q}[S(q, \phi)-1]\}^{2}}{S(q, \phi)\left[D_{S}^{S}(\phi)|\mathbf{k}-\mathbf{q}|^{2}+D^{S}(q, \phi) q^{2}\right]},
\end{aligned}
$$

which can be calculated, once $D_{S}^{S}(\phi), D^{S}(k, \phi)$, and $S(k, \phi)$ are known. We remark that near the glass transition selfconsistent mode-coupling theories are needed and the shorttime expressions (7) and (8) are no longer sufficient to describe the macroscopic transport properties.

From an experimental point of view the long-time wavenumber-dependent self-diffusion coefficient in the limit of $k=0$, i.e., the macroscopic self-diffusion coefficient $D_{S}^{L}(\phi)$ $=D_{S}^{L}(k=0, \phi)$, is particularly important. This quantity is directly related to the long-time mean-square displacement, according to

$$
W(t)=6 D_{S}^{L}(\phi) t, \quad t \gg \tau_{P},
$$

and consequently determines the macroscopic self-diffusion coefficient of a tagged particle as observed in dynamic light scattering experiments. Using Eqs. (24) and (25) for the short-time collective and self-diffusion coefficients, respectively, performing the angular integration and changing to the dimensionless variable $x=q \sigma$, we obtain from Eq. (26) the explicit result for the long-time macroscopic selfdiffusion coefficient,

$$
\frac{D_{S}^{S}(\phi)}{D_{S}^{L}(\phi)}=1+\frac{1}{36 \pi \phi} \int_{0}^{\infty} d x x^{2} \frac{[S(x, \phi)-1]^{2}}{S(x, \phi)+d(x)}
$$

a result previously published by Cohen and de Schepper $[34,35]$.

In Fig. 3, the result (28) for $D_{S}^{L}(\phi)$ is compared with experimental data on neutral colloidal suspensions obtained by several authors [36-38]. For $D_{S}^{S}(\phi)$ the suggestion (25) is used and for $S(k, \phi)$ the same approximation is used as discussed in Sec. III, which compares well with experimental results for $S(k, \phi)$. Also given in Fig. 3 is the result obtained by Medina-Noyola [19], who solved approximately the generalized Langevin equation for the velocity of a tracer particle.

\section{LONG-TIME COLLECTIVE DIFFUSION}

In Sec. II we derived an expression for the the long-time wave-number-dependent collective diffusion coefficient 


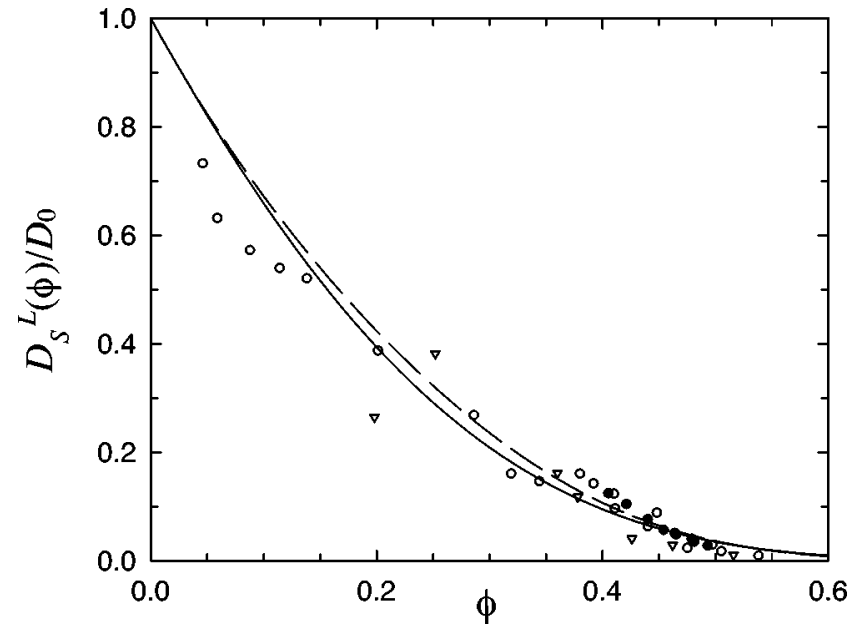

FIG. 3. Relative long-time macroscopic self-diffusion coefficient $D_{S}^{L}(\phi) / D_{0}$ as a function of volume fraction $\phi$. The datapoints are experimental results of neutral PMMA spheres in organic solvents by van Megen et al. [36] (O) and by Segrè et al.. [37] (O), and of neutral silica spheres in cyclohexane obtained by KopsWerkhoven et al. [38] $(\nabla)$. The solid line corresponds to the present theory [cf. Eq. (28)] and the dashed line to that of MedinaNoyola [19], who solved approximately the generalized Langevin equation for the velocity of a tracer particle.

$D^{L}(k, \phi)$ [cf. Eq. (11)]. Restricting ourselves again to first order mode-coupling theory, i.e., using the short-time expression (7) for $F(q, \phi, t)$ and $F(|\mathbf{k}-\mathbf{q}|, \phi, t)$, we obtain the explicit result for $D^{L}(k, \phi)$,

$$
\begin{aligned}
& \frac{D_{S}^{S}(\phi) / S(k, \phi)}{D^{L}(k, \phi)}=1+\frac{1}{16 \pi^{3} n k^{2} D_{S}^{S}(\phi)} \\
& \quad \times \int d \mathbf{q} \frac{[V(k, \mathbf{q})]^{2} / S(q, \phi) S(|\mathbf{k}-\mathbf{q}|, \phi)}{D^{S}(q, \phi) q^{2}+D^{S}(|\mathbf{k}-\mathbf{q}| \phi)|\mathbf{k}-\mathbf{q}|^{2}} .
\end{aligned}
$$

The essential difference of Eq. (29) with Eq. (26), for the long-time self-diffusion coefficient, is the appearance of the unknown three-particle correlation function, $T(\mathbf{k}, \mathbf{q})$, in Eq. (18) for $V(\mathbf{k}, \mathbf{q})$. To evaluate Eq. (29) we use three approximations for $T(\mathbf{k}, \mathbf{q})$ obtained from the literature.

(i) The first approximation was suggested by Hess and Klein [11] and reads

$$
T^{H K}(\mathbf{k}, \mathbf{q})=S(k, \phi)[S(|\mathbf{k}-\mathbf{q}|, \phi)+S(q, \phi)-1] .
$$

Hess and Klein [11] and more recently Wagner [14] applied this approximation to studies of the long-time collective diffusion coefficient of strongly interacting charged colloidal suspensions.

(ii) The second approximation, the so-called convolution approximation, was introduced by Jackson and Feenberg [10] in a study of elementary excitations in liquid helium and reads

$$
T^{J F}(\mathbf{k}, \mathbf{q})=S(k, \phi) S(|\mathbf{k}-\mathbf{q}|, \phi) S(q, \phi) .
$$

This approximation was first used by Szamel and Löwen [13] in an investigation of the glass transition of colloidal suspensions and later by Wagner [14], Baur et al. [15], and Nägele and Baur [16] in studies of charge-stabilized colloidal suspensions.

(iii) The last approximation discussed here is the Kirkwood superposition approximation and reads after a Fourier transformation, using Parcival's theorem and the convolution property of the Fourier transform

$$
\begin{aligned}
T^{K}(\mathbf{k}, \mathbf{q})= & n S(q, \phi) H(k, \phi)+n S(|\mathbf{k}-\mathbf{q}|, \phi) H(q, \phi) \\
& +n S(k, \phi) H(|\mathbf{k}-\mathbf{q}|, \phi)+\frac{n^{2}}{8 \pi^{3}} \\
& \times \int d \mathbf{x} H(|\mathbf{k}-\mathbf{x}|, \phi) H(|\mathbf{q}-\mathbf{x}|, \phi) H(x, \phi)+1,
\end{aligned}
$$

where we have introduced $H(k, \phi)$, the Fourier transform of $h(r, \phi)=g(r, \phi)-1$, i.e.,

$$
H(k, \phi)=\frac{1}{n}[S(k, \phi)-1]
$$

We note that these three approximations do not exhaust the number of approximations found in the literature. However, they are the only closed results in $\mathbf{k}$ space suitable for the calculations that we have performed.

The corresponding expressions for the vertex function $V(\mathbf{k}, \mathbf{q})$ in the Hess and Klein, the Jackson and Feenberg, and the Kirkwood approximation are

$$
\begin{aligned}
V^{H K}(\mathbf{k}, \mathbf{q})= & n D_{S}^{S}(\phi) \mathbf{k} \cdot\{(\mathbf{k}-\mathbf{q}) H(|\mathbf{k}-\mathbf{q}|, \phi)+\mathbf{q} H(q, \phi)\} \\
V^{J F}(\mathbf{k}, \mathbf{q})= & n D_{S}^{S}(\phi) \mathbf{k} \cdot\{(\mathbf{k}-\mathbf{q}) H(|\mathbf{k}-\mathbf{q}|, \phi)+\mathbf{q} H(q, \phi)\} \\
& +n^{2} D_{S}^{S}(\phi) k^{2} H(q, \phi) H(|\mathbf{k}-\mathbf{q}|, \phi)
\end{aligned}
$$

and

$$
\begin{aligned}
V^{K}(\mathbf{k}, \mathbf{q})= & n D_{S}^{S}(\phi) \mathbf{k} \cdot\{(\mathbf{k}-\mathbf{q}) H(|\mathbf{k}-\mathbf{q}|, \phi)+\mathbf{q} H(q, \phi)\} \\
& +\frac{n^{2} D_{S}^{S}(\phi) k^{2}}{S(k, \phi)} H(q, \phi) H(|\mathbf{k}-\mathbf{q}|, \phi) \\
& +\frac{n^{2} D_{S}^{S}(\phi) k^{2}}{S(k, \phi)} \frac{1}{8 \pi^{3}} \int d \mathbf{x} H(|\mathbf{k}-\mathbf{x}|, \phi) \\
& \times H(|\mathbf{q}-\mathbf{x}|, \phi) H(x, \phi),
\end{aligned}
$$

respectively. One can show straightforwardly that all three approximations for $V(\mathbf{k}, \mathbf{q})$ converge in the limit for large $k$ to Eq. (23) for $V_{S}(\mathbf{k}, \mathbf{q})$. Thus, $\lim _{k \rightarrow \infty} D^{L}(k, \phi)$ $=D_{S}^{L}(k, \phi)$, as expected physically.

We have evaluated numerically Eq. (29) for the three approximations as a function of the wave number $k$ for a particular volume fraction $\phi=0.465$. Here, we have used Eqs. (24) and (25) for $D^{S}(k, \phi)$ and $D_{S}^{S}(\phi)$, respectively, and Eq. (33) for $H(k, \phi)$, while $S(k, \phi)$ is calculated as described in Sec. III. The results are given in Fig. 4 and compared to the recent experimental results of Segrè and Pusey [1]. It is clear 


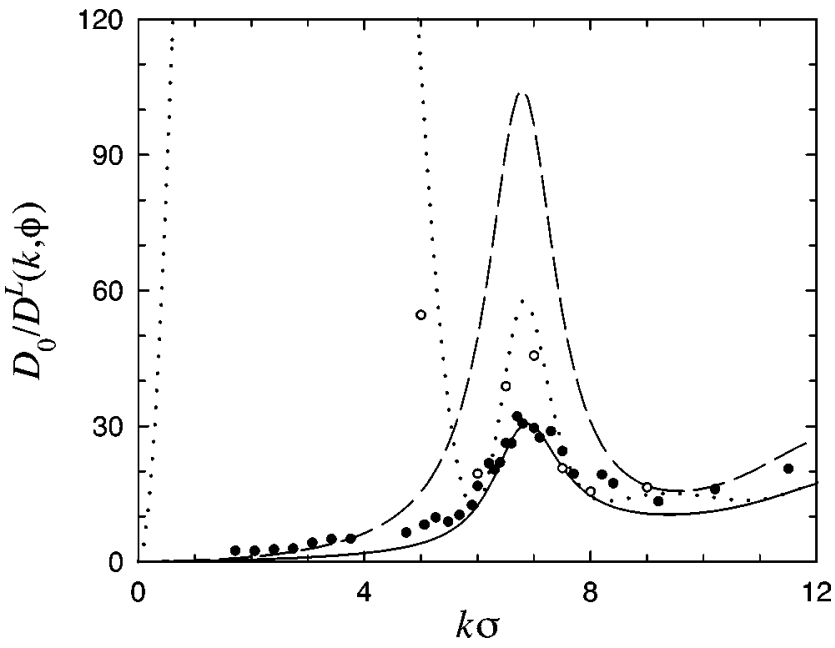

FIG. 4. Inverse relative long-time wave-number-dependent collective diffusion coefficient $D_{0} / D^{L}(k, \phi)$ as a function of $k \sigma$ for various approximations of the three-particle correlation function at $\phi=0.465$. The solid circles are experimental results of neutral PMMA spheres in an organic solvent [1]. The solid line corresponds to the present theory in the convolution approximation [cf. Eqs. (29) and (35)], the dashed line to that in the Hess and Klein approximation [cf. Eqs. (29) and (34)], and the open circles to that in the Kirkwood approximation [cf. Eqs. (29) and (36)]. The dotted line corresponds to the present theory if $T(\mathbf{k}, \mathbf{q})$ is approximated by Eq. (37).

that the Kirkwood approximation is unable to describe $D^{L}(k, \phi)$ for $k \sigma<5$. This is due to the $S(k, \phi)$ in the second term on the right-hand side (rhs) of Eq. (36). For hard spheres and high volume fractions, $S(k, \phi)$ becomes close to zero for a considerable range of $k$ values up to $k \sigma \approx 4$, resulting in a very high value of $V(\mathbf{k}, \mathbf{q})$. This blowing up at small $k$ is absent in the Hess and Klein approximation which is linear in $S(k, \phi)$ [cf. Eq.(30)]. However, this approximation is not symmetric in the mutual exchange of particles and is therefore unphysical. Furthermore, it is approximately a factor 3 too large at intermediate values of $k$.

With respect to symmetry a more physical approximation for the three-particle correlation function on the product level of the static structure factor would be

$$
\begin{aligned}
T(\mathbf{k}, \mathbf{q})= & S(q, \phi)[S(k, \phi)-1]+S(|\mathbf{k}-\mathbf{q}|, \phi)[S(q, \phi)-1] \\
& +S(k, \phi)[S(|\mathbf{k}-\mathbf{q}|, \phi)-1]+1 .
\end{aligned}
$$

Comparing this to the Kirkwood approximation (32) it is clear that this is equivalent to neglecting the integral on the rhs of Eq. (32). The result of this approximation is also presented in Fig. 4. The deviations between this result and the result obtained by using the full Kirkwood approximation are remarkably small and are consequently due to the neglect of the integral on the rhs of Eq. (32). We remark that the result based on Eq. (37) also does not work for $k \sigma<5$, consistent with the fact that it is, just like the Kirkwood approximation, not linear in $S(k, \phi)$.

We found that the best results are obtained by using the convolution approximation (31) of the triple correlation function. In Fig. 5, these results are given for three of the volume fractions for which experimental results were avail-

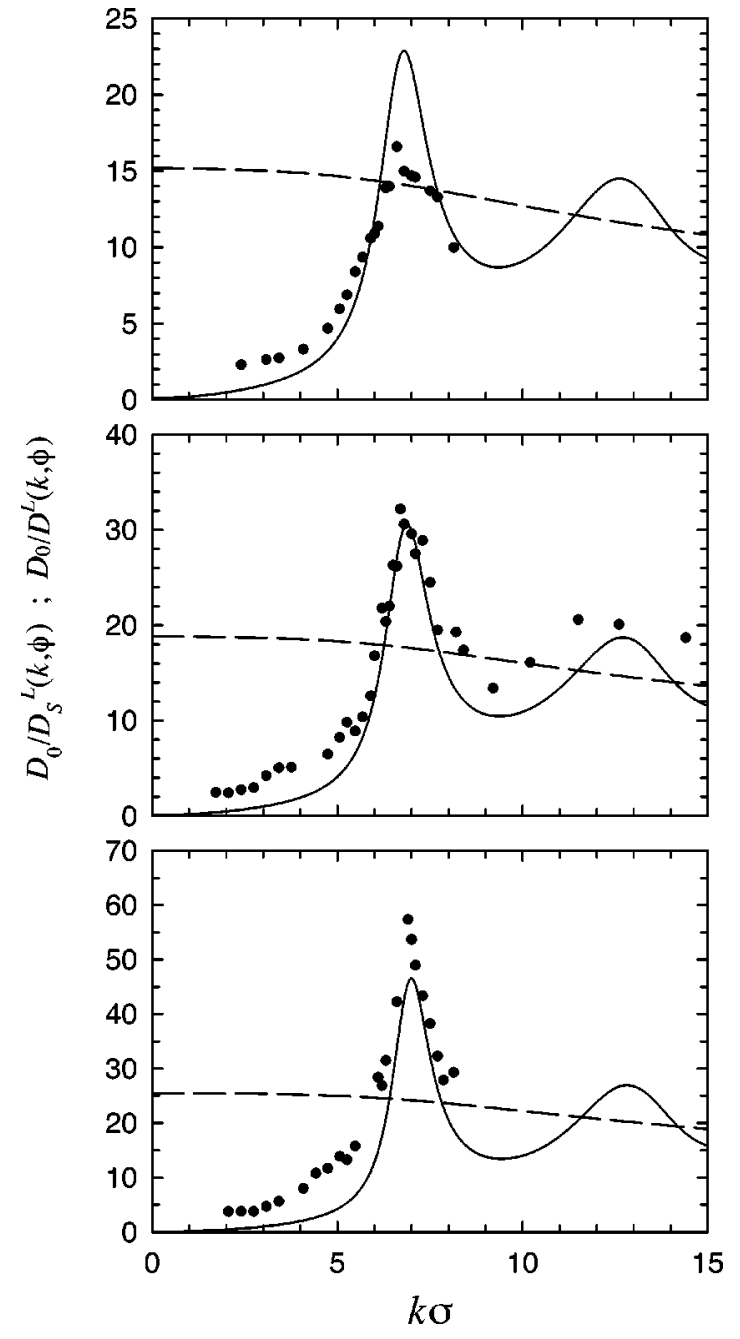

FIG. 5. Inverse relative long-time wave-number-dependent collective diffusion coefficient $D_{0} / D^{L}(k, \phi)$ as a function of $k \sigma$ for three different volume fractions $\phi=0.443$ (a), 0.465 (b), and 0.494 (c). The solid circles are experimental results of neutral PMMA spheres in an organic solvent [1]. The solid line represents the theoretical result in the convolution approximation [cf. Eqs. (29) and (35)]. The dashed line corresponds to the inverse relative long-time wave-number-dependent self-diffusion coefficient $D_{0} / D_{S}^{L}(k, \phi)[\mathrm{cf}$. Eq. (26)].

able. Also given in Fig. 5 is the result for the long-time wave-number-dependent self-diffusion coefficient [cf. Eq. (26)], around which $D^{L}(k, \phi)$ oscillates and to which it converges for large $k$. The intercept of $D_{S}^{L}(k, \phi)$ with the $y$ axis gives the macroscopic self-diffusion coefficient as presented in Fig. 3.

\section{DISCUSSION}

In this paper we have shown that good agreement with experimental results for both the self-diffusion and the collective diffusion can be obtained by using the cage-diffusion process as the primary physical process and incorporating hydrodynamic interaction in a mean-field-like manner. We have shown that recent experimental results, obtained by Segrè and Pusey [1], for the long-time wave-numberdependent collective diffusion coefficient can be well under- 
stood on the basis of the theory presented here, and end with a number of remarks.

(i) The same theory as discussed here has been used before to understand for $k=0$ the dynamic (frequencydependent) and long-time macroscopic (Newtonian) shear viscosity $\eta(\phi, \omega)$ and $\eta_{N}(\phi)$, respectively [2]. Here $\eta_{N}(\phi)$ is given by a time integral like Eq. (22) for the longitudinal viscosity in which $k=0$ and where $\sigma_{\|}(\mathbf{k})$ in Eq. (13) is replaced by the orthogonal component of the microscopic stress [2]. The theoretical $\eta(\phi, \omega)$ and $\eta_{N}(\phi)$ are in good agreement with a large range of experimental data $[2,4]$. Thus it appears that not only the macroscopic $(k=0)$ quantities $\eta(\phi, \omega)$ and $\eta_{N}(\phi)$, but also the long-time wavenumber-dependent diffusion coefficients $D^{\mathrm{L}}(k, \phi)$ and $D_{\mathrm{S}}^{\mathrm{L}}(k, \phi)$ of concentrated neutral colloidal suspensions can be described from one single viewpoint based on a cagediffusion process in the suspension [9].

(ii) The conclusion that for neutral hard-sphere colloidal suspensions the best results for $D^{\mathrm{L}}(k, \phi)$ are obtained by using the convolution approximation (31) of the triple correlation function is consistent with the earlier conclusion of Nägele and Baur [16]. It is interesting to note that we find the same results for concentrated neutral suspensions as they did for dilute but strongly interacting charge-stabilized suspensions.

(iii) During the preparation of this paper Banchio et al. [20] published results for $D^{\mathrm{L}}\left(k=k^{*}, \phi\right), D_{\mathrm{S}}^{\mathrm{L}}(\phi)$ and $\eta_{\mathrm{N}}(\phi)$, based on a self-consistent mode-mode coupling theory. However, in their derivation they completely neglect hydrodynamic interactions, which might well be the reason for the need of two empirical scaling procedures to obtain satisfactory agreement with experiment. First, they scaled the concentration dependence with the volume fraction at the glass transition $\phi_{g}$, i.e., they used an effective volume fraction $\phi_{\text {eff }}=\phi\left(\phi_{g} / 0.525\right)$ to evaluate their results. Here, they used a value $\phi_{g}=0.62$, obtained by fitting their expression for $D_{\mathrm{S}}^{\mathrm{L}}(\phi)$ to Brownian dynamics simulations at high densities. Second, they scaled their mode-coupling expressions in such a way that they effectively obtain the correct limiting behavior for the short-time transport coefficients when the modecoupling contribution to the long-time transport coefficients is neglected, i.e., $D_{\text {eff }}^{\mathrm{L}}\left(k^{*}, \phi\right) \approx H\left(k^{*}, \phi_{\text {eff }}\right) D^{\mathrm{L}}\left(k^{*}, \phi_{\text {eff }}\right)$, $D_{\mathrm{S}, \text { eff }}^{\mathrm{L}}(\phi) \approx D_{\mathrm{S}}^{\mathrm{S}}\left(\phi_{\text {eff }}\right) D_{\mathrm{S}}^{\mathrm{L}}\left(\phi_{\text {eff }}\right) / D_{0} \quad$ and $\quad \eta_{\mathrm{N}, \text { eff }}(\phi)$ $\approx \eta_{\infty}\left(\phi_{\text {eff }}\right) \eta_{\mathrm{N}}\left(\phi_{\text {eff }}\right) / \eta_{0}$. However, although this second scaling gives the correct hydrodynamic behavior at short times, it does not correct for the fact that hydrodynamic interactions also modify the mode-mode coupling contribution to the transport coefficients. This is most likely the reason why they obtain the wrong glass transition, even though they use a self-consistent calculation.

Instead of these scaling rules, we have used a mean-field approximation for the diffusion tensor. We emphasize that this is not a simple scaling with the short-time diffusion coefficients like Bachio et al. [20]. Rather, it consistently modifies both the short-time behavior as well as the modecoupling contribution as is evident by the appearance of $D_{\mathrm{S}}^{\mathrm{S}}(\phi)$ in the denominators of Eqs. (11) and (21) and in the final results for $M(k, \phi, t)$ and $M_{\mathrm{S}}(k, \phi, t)$ [cf. Eqs. (16), (18), (22), and (23)]. Further investigation should focus on finding a better approximation for the diffusion tensor in

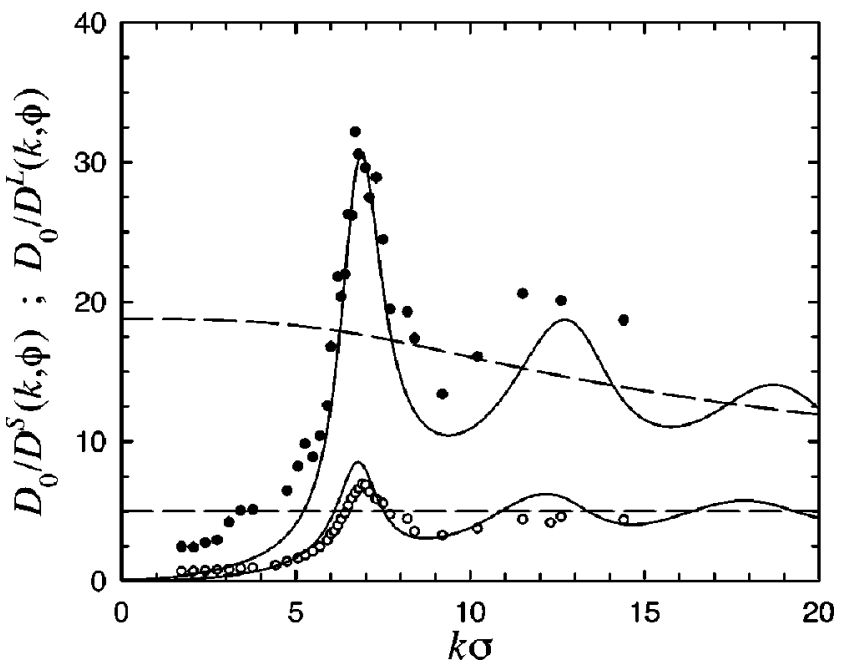

FIG. 6. Inverse relative wave-number-dependent long- and short-time collective diffusion coefficient $D_{0} / D^{L}(k, \phi)$ and $D_{0} / D^{S}(k, \phi)$, respectively, as a function of $k \sigma$ for $\phi=0.465$. The datapoints are experimental results for $D_{0} / D^{L}(k, \phi)(-)$ and for $D_{0} / D^{S}(k, \phi)(\bigcirc)$ of neutral PMMA spheres in an organic solvent [1]. The upper solid line corresponds to the present theory for $D_{0} / D^{L}(k, \phi)$ in the convolution approximation [cf. Eqs. (29) and (35)], the lower solid line to that for $D_{0} / D^{S}(k, \phi)$ [cf. Eq. (24)], the upper dashed line corresponds to that for $D_{0} / D_{S}^{L}(k, \phi)$ [cf. Eq. (26)] and finally the lower dashed line to that for $D_{0} / D_{S}^{S}(\phi)$ [cf. Eq. (25)].

order to incorporate the near-field hydrodynamic interactions, which dominate the interaction at short times in neutral hard-sphere colloidal suspensions.

(iv) The shape of the theoretical result for $D_{0} / D^{\mathrm{L}}(k, \phi)$ as a function of $k$ is very similar to that of $D_{0} / D^{\mathrm{S}}(k, \phi)$ for all $\phi$, a result already noted by Sergè and Pusey [1]. As shown in Fig. $6, D^{\mathrm{L}}(k, \phi)$ as a function of $k$ oscillates around $D_{\mathrm{S}}^{\mathrm{L}}(k, \phi)$, very much like $D^{\mathrm{S}}(k, \phi)$ oscillates around $D_{\mathrm{S}}^{\mathrm{S}}(\phi)$. Therefore, the ratio $D^{\mathrm{L}}(k, \phi) / D^{\mathrm{S}}(k, \phi)$ is mainly determined by the ratio $D_{\mathrm{S}}^{\mathrm{L}}(k, \phi) / D_{\mathrm{S}}^{\mathrm{S}}(\phi)$ as a function of $k$ and $\phi$. For $\phi=0.465$ and $k \sigma$ near $k^{*} \sigma \approx 2 \pi$ this ratio is approximately independent of $k$ and equal to 3.6, close to the value of 4.5 found by Segrè and Pusey [1].

We note that this scaling result only holds for $k$ values near $k^{*}$, i.e., near the first peak of the static structure factor, since for monodisperse suspensions the ratio $D^{L}(k, \phi) / D^{\mathrm{S}}(k, \phi)$ tends to one for the both $k \rightarrow 0$ as well as for $k \rightarrow \infty$. For $k \rightarrow 0$ this is due to the fact that the plane wave collective density fluctuation $\delta n(\mathbf{k})$ of Eq. (2) becomes an exact eigenfunction of the Smoluchowski operator $\hat{\Omega}$ for $k \rightarrow 0$, with a single eigenvalue $D^{\mathrm{L}}(k=0, \phi)=D_{\mathrm{S}}^{\mathrm{L}}(k=0, \phi)$. It appears that $D^{\mathrm{L}}(k, \phi) / D^{\mathrm{S}}(k, \phi)$ quickly drops from 1 at $k=0$ to a value characterized by $D_{\mathrm{S}}^{\mathrm{L}}(k, \phi) / D_{\mathrm{S}}^{\mathrm{S}}(\phi)$ when $k \sigma$ $>4$.

(v) Figure 5 clearly shows that the theory predicts for $k \sigma<5$ a larger value of $D^{\mathrm{L}}(k, \phi)$ than was found experimentally, for all volume fractions. Furthermore, it shows that for small values of $k \sigma$, the experimental values for $D^{\mathrm{L}}(k, \phi)$ are considerably smaller than those for $D^{\mathrm{S}}(k, \phi)$, in contradiction with the above mentioned exact result for monodisperse suspensions, i.e., $D^{\mathrm{L}}(k=0, \phi) / D^{\mathrm{S}}(k=0, \phi)=1$. This suggests that the deviation is caused by the polydispersity of the 


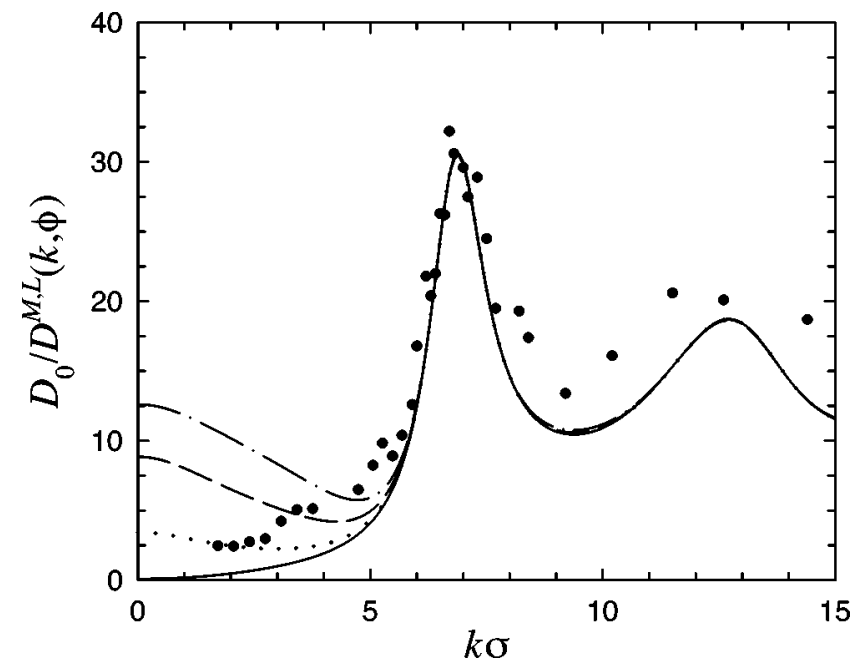

FIG. 7. Inverse relative long-time wave-number-dependent collective diffusion coefficient $D_{0} / D^{M, L}(k, \phi)$ in the convolution approximation [cf. Eq. (39)] as a function of $k \sigma$ for $\phi=0.465$ and for different polydispersities $s=2$ (dotted line), 4 (dashed line), and 6 (dash-dotted line) compared to the experimental results of a suspension of PMMA spheres in cis-decalin ( ), with a polydispersity of $s \approx 5$ [1]. The solid line corresponds to a monodisperse suspension [cf. Fig. 5(b)].

PMMA particles. In fact, in order to study the effect of a small polydispersity on the long-time wave-numberdependent collective diffusion coefficient we have calculated $D^{\mathrm{L}}(k, \phi)$ for a suspension of hard-sphere particles with a narrow size distribution with a standard deviation $s \ll 1$ around a mean diameter $\langle\sigma\rangle$, by replacing the intermediate scattering function $F(k, \phi, t)$ in Eq. (9) with

$$
F^{\mathrm{M}}(k, \phi, t)=(1-x) F(k, \phi, t)+x F_{\mathrm{S}}(k, \phi, t) .
$$

Here $F(k, \phi, t)\left[F_{\mathrm{S}}(k, \phi, t)\right]$ is the intermediate [self-] scattering function of the monodisperse suspension and $x=1$ $-\left\langle\sigma^{3}\right\rangle^{2} /\left\langle\sigma^{6}\right\rangle \approx 9 s^{2}$. Strictly, this approximation is only valid for suspensions of particles with equal diameter and interaction potential, but with different scattering amplitude, but the same decoupling is likely to hold for a small degree of size polydispersity as well [21].

Following then essentially the same procedure in Refs. $[15,16]$ for charged colloids, the following result is obtained for the measured long-time wave-number-dependent collective diffusion coefficient $D^{\mathrm{M}, \mathrm{L}}(k, \phi)$ of a neutral hardsphere-like colloidal suspension with a small size polydispersity

$$
\begin{aligned}
D^{\mathrm{M}, \mathrm{L}}(k, \phi)= & D^{\mathrm{L}}(k, \phi) \\
& \times\left\{\frac{S^{\mathrm{M}}(k, \phi)}{(1-x) S(k, \phi)+x D^{\mathrm{L}}(k, \phi) / D_{\mathrm{S}}^{\mathrm{L}}(k, \phi)}\right\},
\end{aligned}
$$

where $S^{\mathrm{M}}(k, \phi)=F^{\mathrm{M}}(k, \phi, t=0)$, the measured equilibrium static structure factor. Figure 7 shows the result of Eq. (39) for $\phi=0.465$, for different polydispersities $s=0.02,0.04$, and 0.06 , compared with the experimental result obtained by Segrè and Pusey [1] on a suspension of PMMA spheres in cis-decalin, with a polydispersity of 0.05 . The decoupling approximation predicts the observed reduction (increase) of $D^{\mathrm{L}}(k, \phi) / D_{0}\left[D_{0} / D^{\mathrm{L}}(k, \phi)\right]$, at small values of $k \sigma$, that was found experimentally, but it seems to overestimate the effect. This may well be due to the crude approximation of Eq. (38) and consequently the very approximate nature of Eq. (39). However, the qualitative agreement supports the hypothesis that the deviation between theory and experiment at small values of $k \sigma$ is caused by the polydispersity of the colloidal particles confirming the same conclusion reached before for charged suspensions $[15,16]$.

(vi) We have computed the time-correlation functions $M(k, \phi, t)$ of Eq. (16) and $M_{\mathrm{S}}(k, \phi, t)$ of Eq. (22) for all times using the short-time expressions for $F(k, \phi, t)$ and $F_{\mathrm{S}}(k, \phi, t)$ given by Eqs. (7) and (8), respectively. This inconsistency can be remedied by using a self-consistent theory, where the behavior of $F(k, \phi, t)$ and $F_{\mathrm{S}}(k, \phi, t)$ is consistently modified in concordance with the computation of the long-time diffusion coefficients $D^{\mathrm{L}}(k, \phi)$ and $D_{\mathrm{S}}^{\mathrm{L}}(k, \phi)$, respectively. This is done in calculations for undercooled fluids and the glass transition [39] and more recently in calculations for concentrated charged-stabilized colloidal suspensions [16]. Furthermore, we note that our expression (24) is strictly valid only for wave numbers near $k=k^{*}$ (although Fig. 1 shows that it compares well with experiment for a considerable range of $k$ values), where the relaxation is particularly slow. For wave numbers farther away from the peak of the static structure factor the decay of the intermediate scattering function becomes nonexponential at intermediate and long times [40] and the use of the shorttime expression (7) for $F(k, \phi, t)$, with $D^{\mathrm{S}}(k, \phi)$ given by Eq. (24) is once again no longer sufficient.

In addition, the Carnahan-Starling approximation used for $\chi(\phi)$ in Eq. (25) may not be adequate for the large volume fractions $\phi$ used in Segrè and Pusey's experiments which approach the freezing transition. It seems that at these very high volume fractions the theory is not fully able to describe the rapid decrease of $D^{L}(k, \phi)$ at wave number near $k$ $=k^{*}$. This was also observed in a study of the Newtonian viscosity of concentrated neutral colloidal suspensions [4], where a quantitative agreement could be obtained after the radial distribution function at contact $\chi(\phi)=g(r=\sigma, \phi)$ was replaced by an expression with a pole near the glass transition. This one-pole approximation, suggested by Brady [23], seems be more appropriate at the highest volume fraction and would increase the theoretical maxima near $k=k^{*}$ in the direction of the experimental data.

However, in view of the many approximations made in the theory and the possible systematic errors in the experimental results, it is not easy to ascertain the origin of the deviations between theory and experiment. Nevertheless, the overall agreement and similarity in behavior suggests that the basic physical effects in concentrated colloidal suspensions are present in the theory presented here.

\section{ACKNOWLEDGMENTS}

R.V. gratefully acknowledges financial support from the Netherlands Foundation for Fundamental Research of Matter (Stichting FOM), and E.G.D.C. from the DOE under Grant No. DE-FG02-88-ER13847. 
[1] P. N. Segrè and P. N. Pusey, Phys. Rev. Lett. 77, 771 (1996).

[2] R. Verberg, E. G. D. Cohen, and I. M. de Schepper, Phys. Rev. E 55, 3143 (1997).

[3] E. G. D. Cohen, R. Verberg, and I. M. de Schepper, Int. J. Multiphase Flow 23, 797 (1997).

[4] E. G. D. Cohen, R. Verberg, and I. M. de Schepper, Physica A 251, 251 (1998).

[5] E. G. D. Cohen and I. M. de Schepper, J. Stat. Phys. 63, 241 (1991).

[6] I. M. de Schepper, E. G. D. Cohen, and M. J. Zuilhof, Phys. Lett. 101A, 399 (1984).

[7] E. G. D. Cohen, I. M. de Schepper, and M. J. Zuilhof, Physica B \& C 127, 282 (1984).

[8] I. M. de Schepper, E. G. D. Cohen, P. N. Pusey, and H. N. W. Lekkerkerker, J. Phys.: Condens. Matter 1, 6503 (1989).

[9] R. Verberg, Ph.D. thesis, Delft University of Technology, 1998.

[10] H. W. Jackson and E. Feenberg, Rev. Mod. Phys. 34, 686 (1962).

[11] W. Hess and R. Klein, Adv. Phys. 32, 173 (1983).

[12] B. Cichocki and W. Hess, Physica A 141, 475 (1987).

[13] G. Szamel and H. Löwen, Phys. Rev. A 44, 8215 (1991).

[14] N. J. Wagner, Phys. Rev. E 49, 376 (1994).

[15] P. Baur, G. Nägele, and R. Klein, Phys. Rev. E 53, 6224 (1996)

[16] G. Nägele and P. Baur, Physica A 245, 297 (1997).

[17] G. Nägele and J. K. G. Dhont, J. Chem. Phys. 108, 9566 (1999).

[18] G. Nägele, J. Bergebholtz, and J. K. G. Dhont, J. Chem. Phys. 110, 7037 (1999).

[19] M. Medina-Noyola, Phys. Rev. Lett. 60, 2705 (1988).

[20] A. J. Banchio, J. Bergenholtz, and G. Nägele, Phys. Rev. Lett. 82, 1792 (1999).

[21] P. N. Pusey and R. J. A. Tough, in Dynamic Light Scattering, edited by R. Pecora (Plenum Press, New York, 1985), pp. 85-179.

[22] J. K. W. Dhont, An Introduction of Dynamics of Colloids (Elsevier, Amsterdam, 1996).

[23] J. F. Brady, J. Chem. Phys. 99, 567 (1993).

[24] G. K. Batchelor, J. Fluid Mech. 74, 1 (1976).

[25] B. Cichocki and B. U. Felderhof, J. Chem. Phys. 89, 1049 (1988)
[26] C. W. J. Beenakker and P. Mazur, Physica A 126, 349 (1984).

[27] A. J. C. Ladd, J. Chem. Phys. 93, 3484 (1990).

[28] E. G. D. Cohen and I. M. de Schepper, Phys. Rev. Lett. 75, 2252 (1995).

[29] D. Henderson and E. W. Grundke, J. Chem. Phys. 63, 601 (1975).

[30] W. van Megen, R. H. Ottewil, S. M. Owens, and P. N. Pusey, J. Chem. Phys. 82, 508 (1985).

[31] I. M. de Schepper, E. G. D. Cohen, and R. Verberg, Phys. Rev. Lett. 77, 584 (1996).

[32] P. N. Segrè, S. P. Meeker, P. N. Pusey, and W. C. K. Poon, Phys. Rev. Lett. 77, 585 (1996).

[33] I. M. de Schepper, R. Verberg, and E. G. D. Cohen, Phys. Rev. Lett. 77, 584 (1996).

[34] E. G. D. Cohen and I. M. de Schepper, in Recent Progress in Many-Body Theories 3, edited by T. L. Ainsworth, C. E. Campbell, B. E. Clements, and E. Krotscheck (Plenum, New York, 1992), pp. 387-395.

[35] E. G. D. Cohen and I. M. de Schepper, in Slow Dynamics in Condensed Matter, Proceedings of the First Tohwa University International Symposium, Fukuoka, Japan, 1991, edited by K. Kawasaki, T. Kawakatsu, and M. Tokuyama, AIP Conf. Proc. No. 256 (AIP, Woodbury, NY, 1992), pp. 359-369.

[36] W. van Megen et al., Faraday Discuss. Chem. Soc. 83, 47 (1987).

[37] P. N. Segrè, S. P. Meeker, P. N. Pusey, and W. C. K. Poon, Phys. Rev. Lett. 75, 958 (1995).

[38] M. M. Kops-Werkhoven and H. M. Fijnaut, J. Chem. Phys. 77, 2242 (1982).

[39] W. Götze, in Liquids, Freezing and the Glass Transition, edited by J. P. Hansen, D. Levesque, and J. Zinn-Justin (NorthHolland, Amsterdam, 1991), pp. 287-503.

[40] B. Cichocki and B. U. Felderhof, Physica A 204, 152 (1994).

[41] P. N. Segrè, O. P. Behrend, and P. N. Pusey, Phys. Rev. E 52, 5070 (1995).

[42] P. N. Pusey and W. van Megen, Phys. Rev. Lett. 59, 2083 (1987).

[43] J. X. Zhu et al., Phys. Rev. Lett. 68, 2559 (1992).

[44] P. N. Pusey and W. van Megen, J. Phys. (France) 44, 285 (1983).

[45] A. van Veluwen, H. N. W. Lekkerkerker, C. G. de Kruif, and A. Vrij, J. Chem. Phys. 87, 4873 (1987).

[46] A. J. C. Ladd, Phys. Rev. Lett. 70, 1339 (1993). 